\title{
A influência do padrão de aleitamento no desenvolvimento de hábitos de sucção não nutritivos na primeira infância
}

\author{
The influence of feeding methods in the development \\ of nonnutritive sucking habits in childhood
}

Sabrina Sales Lins de Albuquerque ${ }^{1}$

Ricardo Cavalcanti Duarte ${ }^{1}$

Alessandro Leite Cavalcanti ${ }^{2}$

Érika deM orais Beltrão ${ }^{1}$

${ }^{1}$ Departamento deClínicae OdontologiaSocial, FaculdadedeOdontologia, UniversidadeFederal da Paraíba. Cidade Universitária. 58039-900 João Pessoa PB. sabrina.lins@bol.com.br

${ }^{2}$ Departamento de Odontologia, Universidade Estadual da Paraíba.
Abstract This study verified the relationship between nonnutritive sucking habits and feeding methods in children from 12 to 36 months of age, attending public nursery schools in the city of João Pessoa, Paraíba State. The sample consisted of 292 children of both gender and the data were collected by interviewing the children's mothers or minders. The information of the forms consisted on the description of the frequency and duration of the nonnutritive sucking habits, as well as on the feeding methods. The data were analyzed through the statistical program SPSS, taking place distributions of frequencies and quisquare test and Fisher Exact. In relation to the presence of nonnutritive sucking habits, $69.2 \%$ of the children had sometype of habit, being $61.6 \%$ the pacifier sucking and $8.2 \%$ the digital sucking. From the entire sample, $10.2 \%$ presented exclusive breastfeeding, $4.9 \%$ were just bottle-fed and $84.9 \%$ were breast-fed and bottle-fed. Considering the methods of feeding, $83.8 \%$ of the children presented exclusive breastfeeding during the first 6 months of life. The feeding methods presented significant association with the presence of nonnutritive sucking habits. As larger the duration of the exclusive breastfeeding, smaller the prevalence of sucking habits. Key words Breastfeeding, Sucking habits, Bottle feeding
Resumo 0 estudo analisou a relação entre o padrão de alei tamento e o desenvolvimento de hábitos de sucção não-nutritivos em crianças de 12 a 36 meses deidade, de creches públicas na cidadede João Pessoa (PB). A amostra constou de 292 crianças, deambos os gêneros ea coleta de dados abrangeu entrevistas com asmães/responsáveis. Asinformações dos formulários consistiam na descrição da frequência eduração dos hábitos desucção nãonutritivos, bem como dos métodos de aleitamento. Os dados foram analisados através do programa SPSS, realizando-se distribuições de frequências e os testes estatísticos do qui-quadrado e Exato de Fisher. O bservou-se que, com relação à presença de hábitos de sucção não-nutritivos, $69,2 \%$ das criançastinham algum tipo dehábito, sendo $61,6 \%$ a sucção de chupeta e $8,2 \%$ a sucção digital. De toda a amostra, $10,2 \%$ realizaram aleitamento natural exclusivo, 4,9\%, 0 aleitamento artificial exclusivo e $84,9 \%, 0$ aleitamento misto. Considerando os tipos de aleitamento, 83,8\% das crianças realizaram aleitamento natural exclusivo durante os seis primeiros meses de vida. 0 padrão de aleitamento apresentou associação significativa com a presença de hábitos de sucção não-nutritivos. Quanto maior a duração do aleitamento natural exclusivo, menor a prevalência de hábitos de sucção não-nutritivos e o padrão de aleitamento. Palavras-chave Aleitamento materno, Hábitos de sucção, Alimentação artificial 
Introdução

Nas últimas décadas, informações sobre as vantagens do aleitamento materno têm encorajado esforços para reverter a tendência do desmame precoce, condição esta considerada biológica e medicamente regressiva pelos pediatras e nutricionistas ${ }^{1}$. 0 aleitamento materno deve ser exclusivo até os seis meses de idade e complementado até dois anos ou mais².

0 bebê apresenta, desde 0 nascimento, uma necessidade inerenteà real ização da sucção, a qual pode ser satisfeita de duas formas: nutritiva e não-nutritiva. A primeira fornece os nutrientes alimentares, através do aleitamento natural ou artificial, enquanto a segunda proporciona à criança prazer especial, sensação de bem-estar e proteção, satisfazendo-a psicologicamente ${ }^{3}$. Os exemplos mais comuns dehábitos de sucção nãonutritivos são a sucção digital e de chupeta e o prolongamento destes hábitos pode ter consequências nocivas ao desenvolvimento do sistema estomatognático ${ }^{4-6}$.

Os hábitos de sucção não-nutritivos são relatados como um dos mais importantes causadores de alterações oclusais ${ }^{7-10}$. A literatura cita como exemplos de agentes causais: crianças que não tiveram acesso à amamentação natural irrestrita, quetiveram desmame prematuro ou que foram alimentadas com a mamadeira ${ }^{3,11}$.

0 aleitamento natural, além de prover todas as necessidades nutricionais do lactente, nos primeiros meses de vida, proporciona, pelo contato físico entrea mãeefilho, interações benéficas entre ambos, que se constituirão no embasamento da formação da concepção adequada do mundo da criança ${ }^{12}$. A presenta ainda ação benéfica na prevenção da instalação e persistência dos hábitos de sucção não-nutritivos ${ }^{13}$.

Crianças com menor tempo de aleitamento materno desenvolvem, com maior frequência, hábitos bucais deletérios, possuindo o risco relativo sete vezes superior àquelas aleitadas no seio por um período de, no mínimo, seis meses. As crianças aleitadas com mamadeira por mais de um ano apresentam quase dez vezes mais risco de apresentarem hábitos bucais viciosos do que aquelas que nunca utilizaram essa forma de aleitamento ${ }^{12}$.

Alguns hábitos agem como estímulos ao crescimento normal dos maxilares, favorecendo a liberação do potencial de crescimento facial em sua plenitude, sem desvios, onde se incluem a respiração nasal, a deglutição, a mastigação, a fonação e a posição adequada da língua e dos lábios ${ }^{11}$. Outros hábitos estão associados a alterações do crescimento ósseo, más posições dentárias e dificuldade na fala. Dentro deste contexto, estão os hábitos de sucção digital e de chupeta, a respiração bucal, as funções anormais da língua durante a deglutição, fonação e postura, dentre outros ${ }^{14}$.

Diante da escassez de estudos que analisam a influência do padrão de aleitamento no desenvolvimento de hábitos de sucção não-nutritivos em crianças de pouca idade, na cidade de João Pessoa (PB), este estudo transversal propôs-se a verificar esta associação em crianças de 12 a 36 meses de idade, matriculadas em creches públicasnestemunicípio.

\section{Métodos}

Esta pesquisa foi aprovada pelo Comitê de Ética em Pesquisa do Centro de Ciências da Saúde da Universidade Federal da Paraíba, de acordo com a Resolução no 196/96 do CN S/M S que regulamenta a pesquisa em seres humanos.

0 estudo foi desenvolvido em creches públicas na cidade de João Pessoa (PB), sendo quinze creches municipais etreze creches estaduais, onde foram avaliadas 292 crianças na faixa etária de 12 a 36 meses, das quais 62 crianças tinham de 4 a 24 meses e 230, de 24,1 a 36 meses de idade, de ambos os gêneros (161 meninos - 55,1\% - e 131 meninas $-44,9 \%$ ), regularmente matriculadas nesses estabelecimentos no ano de 2003. A escoIha dos locais deu-se através da técnica de amostragem aleatória simples.

A coleta de dados foi realizada após a autorização dos pais e/ou responsáveis das crianças participantes, os quais foram devidamente informados do propósito do estudo. Com relação à identificação das crianças examinadas, esta foi obtida através dos prontuários existentes nas instituições selecionadas, onde se registrou 0 nome e a data de nascimento das crianças.

A presença ou ausência de hábitos de sucção não-nutritivos, tipo de hábito, frequência e duração e o padrão de aleitamento foram avaliados por meio de formulários aplicados aos pais e/ou responsáveis, no horário de entrada e saída das crianças ou durante as reuniões de pais realizadas nas creches.

Com relação à presença do hábito, questionou-se os pais/responsáveis se a criança possuía o hábito de sucção de dedo e/ou de chupeta, sendo registrado a duração do mesmo, ou seja, período em que o hábito havia sido iniciado e até 
que idade prolongou-se. A frequência do hábito de sucção não-nutritivo presente foi classificada em diurno e noturno.

0 tipo do bico da chupeta foi classificado em tradicional eortodôntico. As dúvidas dos pais em relação a estas nomenclaturas foram resolvidas pelas pesquisadoras no momento da entrevista.

Questionou-se, também, o tipo dealeitamento que a criança realizou, ou seja, aleitamento materno exclusivo, aleitamento artificial exclusivo ou aleitamento misto, no qual a criança foi aleitada no seio eatravés da mamadeira, eo tempo deste aleitamento.

Considerou-secomo aleitamento natural exclusivo quando a criança alimentava-se apenas no seio materno. 0 aleitamento artificial exclusivo foi verificado quando a criança alimentava-se apenas através do uso de mamadeira. 0 tempo de aleitamento natural foi considerado exclusivo, ou seja, atéqueidade a criança não fez uso de alimentação complementar, e total, ou seja, até que idade a criança utilizou al eitamento natural associado à alimentação complementar.

Para a análise dos dados, foram obtidas as frequências absolutas e percentuais (técnicas de estatística descritiva) eutilizadas o testequi-quadrado de independência de Pearson eo teste Exato de Fisher quando as condições para utilização do qui-quadrado não foram verificadas. $\mathrm{N}$ as tabelas bivariadas foi também apresentado 0 valor do odds ratio (OR) e um intervalo de confiança para esta medida.

O nível designificânciautilizado nas decisões dos testes estatísticos foi de 5\%. Os dados foram digitados na planilha Excel e os softwares utilizados para a obtenção dos cál culos estatísticos foram o SPSS (Statistical Package for the Social Sciences) na versão 11 e SAS (Statistical Analysis System) na versão 8.

\section{Resultados}

Com relação à presença de hábito de sucção, verificou-se que 202 crianças $(69,2 \%)$ possuíam algum tipo de hábito de sucção não-nutritivo, com a maioria (91,0\%) tendo iniciado estehábito no período desdeo nascimento atéos três primeiros meses de vida. Na Tabela 1, é possível verificar que $61,6 \%$ das crianças apresentavam 0 hábito de sucção de chupeta.

Entre as crianças que possuíam o hábito de sucção de chupeta, $60,0 \%$ permaneceram com 0 hábito até a idade de 25 a 36 meses (Tabela 2). Observa-se ainda que apenas $36,1 \%$ faziam uso da chupeta no período noturno e que a chupeta do tipo tradicional foi a mais comumente utilizada $(81,1 \%)$.

Apenas $10,2 \%$ da amostrativeram al eitamento exclusivo conforme pode ser verificado na Tabela 3. Em relação ao tempo de aleitamento natural total, a maior frequência foi para o tempo de 12 a 24 meses, correspondendo a $48,3 \%$.

Tabela 1. Distribuição das crianças segundo os tipos de hábitos de sucção não-nutritivos. João Pessoa (PB).

\begin{tabular}{|c|c|c|c|c|c|c|}
\hline \multirow{3}{*}{$\begin{array}{l}\text { Tipos de hábitos } \\
\text { de sucção } \\
\text { não-nutritivos }\end{array}$} & \multicolumn{4}{|c|}{ Presente } & & \\
\hline & \multicolumn{2}{|c|}{ Sim } & \multicolumn{2}{|c|}{ Não } & \multicolumn{2}{|c|}{ Total } \\
\hline & $n$ & $\%$ & $n$ & $\%$ & $n$ & $\%$ \\
\hline Sucção digital & 24 & 8,2 & 268 & 91,8 & 292 & 100,0 \\
\hline Sucção de chupeta & 180 & 61,6 & 112 & 38,4 & 292 & 100,0 \\
\hline
\end{tabular}

(1) Destaca-seque duas crianças tinham ambos os hábitos.

Tabela 2. Avaliação da duração e frequência da sucção digital e da sucção de chupeta e tipo de chupeta utilizado pelas crianças pesquisadas. João Pessoa (PB).

\begin{tabular}{|c|c|c|}
\hline Variáveis & $\mathrm{N}$ & $\%$ \\
\hline \multicolumn{3}{|c|}{ D uração da sucção digital } \\
\hline Até 12 meses & 2 & 8,3 \\
\hline De 13 a 24 meses & 7 & 29,2 \\
\hline De 25 a 36 meses & 15 & 62,5 \\
\hline Total & 24 & 100,0 \\
\hline \multicolumn{3}{|c|}{ Frequência da sucção digital } \\
\hline Noturno & 11 & 45,8 \\
\hline Diurno e noturno & 13 & 54,2 \\
\hline Total & 24 & 100,0 \\
\hline \multicolumn{3}{|c|}{ Duração da sucção de chupeta } \\
\hline Até 12 meses & 31 & 17,2 \\
\hline De 13 a 24 meses & 41 & 22,8 \\
\hline De 25 a 36 meses & 108 & 60,0 \\
\hline Total & 180 & 100,0 \\
\hline \multicolumn{3}{|c|}{ Frequência da sucção de chupeta } \\
\hline Diurno e noturno & 111 & 61,7 \\
\hline Noturno & 65 & 36,1 \\
\hline Outros & 4 & 2,2 \\
\hline Total & 180 & 100,0 \\
\hline \multicolumn{3}{|l|}{ Tipo de chupeta } \\
\hline Tradicional & 146 & 81,1 \\
\hline Ortodôntica & 32 & 17,8 \\
\hline Ambas & 2 & 1,1 \\
\hline Total & 180 & 100,0 \\
\hline
\end{tabular}


Tabela 3. Tempos de aleitamento apresentados pelas crianças pesquisadas. João Pessoa (PB).

\begin{tabular}{|c|c|c|c|}
\hline Grupos & Tempo em meses & $\mathrm{N}$ & $\%$ \\
\hline \multirow{9}{*}{$\begin{array}{l}\text { Aleitamento natural exclusivo } \\
(10,2 \%, n=29)\end{array}$} & Aleitamento natural exclusivo & & \\
\hline & Até 6 & 18 & 62,1 \\
\hline & M ais de 6 até 12 & 5 & 17,2 \\
\hline & M ais de 12 a 24 & 6 & 20,7 \\
\hline & Aleitamento natural total & & \\
\hline & Até 6 & 4 & 13,8 \\
\hline & Mais de 6 até 12 & 2 & 6,9 \\
\hline & Mais de 12 a 24 & 14 & 48,3 \\
\hline & Mais de 24 a 36 & 9 & 31,0 \\
\hline \multirow{5}{*}{$\begin{array}{l}\text { Aleitamento artificial exclusivo } \\
(4,9 \%, n=14)\end{array}$} & Aleitamento artificial & & \\
\hline & Até 6 & 1 & 7,1 \\
\hline & M ais de 6 até 12 & 1 & 7,1 \\
\hline & M ais de 12 a 24 & 4 & 28,6 \\
\hline & M ais de 24 a 36 & 8 & 57,1 \\
\hline \multirow{14}{*}{$\begin{array}{l}\text { Aleitamento misto }{ }^{1} \\
(84,9 \%, n=242)\end{array}$} & Aleitamento natural exclusivo & & \\
\hline & Até 6 & 207 & 83,1 \\
\hline & M ais de 6 até 12 & 29 & 11,6 \\
\hline & Mais de 12 a 24 & 6 & 2,4 \\
\hline & Aleitamento natural total & & \\
\hline & Até 6 & 164 & 65,9 \\
\hline & Mais de 6 até 12 & 35 & 14,1 \\
\hline & M ais de 12 a 24 & 28 & 11,2 \\
\hline & M ais de 24 a 36 & 15 & 6,0 \\
\hline & Aleitamento artificial & & \\
\hline & Até 6 & 7 & 2,8 \\
\hline & M ais de 6 até 12 & 32 & 12,9 \\
\hline & Mais de 12 a 24 & 88 & 35,3 \\
\hline & M ais de 24 a 36 & 115 & 46,2 \\
\hline
\end{tabular}

(1) Para sete pesquisados, não se dispõe das informações sobre amamentação.

Entreas crianças com al eitamento misto (84,9\%), o maior percentual de crianças com aleitamento natural exclusivo ealeitamento natural total ocorreu no intervalo até 6 meses - 83,1\% 365,9\%, respectivamente.

Entre as crianças com aleitamento natural exclusivo, a maioria ( $82,8 \%$ ) não possuía nenhum tipo de hábito de sucção não-nutritivo (Tabela 4). Constata-se ainda que as crianças que prolongaram o aleitamento natural total além dos seis meses tiveram, progressivamente, até os 36 meses, menos hábitos de sucção não-nutritivos.

O percentual da ocorrência dehábitos desucção não-nutritivos diminuiu à medida queo tempo de aleitamento natural exclusivo aumentou, variando de $79,3 \%$ na faixa até seis meses para 33,3\% para a faixa de mais de 12 a 24 meses (Tabela5). Encontrou-seassociação positiva entreas faixas de tempo de al eitamento e a ocorrência de hábitos de sucção não nutritivos ( $p<0,001$ ).

\section{Discussão}

O comportamento de sucção nos bebês e crianças pequenas é derivado da necessidade fisiológica denutrientes. Estudos sobreo desenvolvimento infantil sugerem que a sucção também aumenta e continua devido a necessidades psicológicas. Desta forma, bebês normalmente desenvolvidos têm tendência inerentee biológica à sucção ${ }^{15}$. A manutenção deste reflexo até os 18 a 24 meses é considerado normal11,16. M as, além deste período, transforma-se em um mau hábito, já que nessa idade encontram-se quase todos os elementos dentários decíduos na boca e a sucção 
se faz desnecessária, sendo substituída, progressivamente, pelo reflexo de apreensão ou pelo impulso de morder, definindo-se o padrão mastigatório ${ }^{11}$, concomitante à evolução do aprendizado da fala ${ }^{14}$. Portanto, passam a ser considerados deletérios após os quatro anos de idade, pois levam a distúrbios do desenvolvimento normal da oclusão e da face ${ }^{17}$.

A influência do padrão deal eitamento na pre sença de hábitos de sucção não- nutritivos foi avaliada por diversos autores ${ }^{13,18-23}$, sendo observado que quanto maior o tempo de aleitamento natural, menor o percentual de crianças que apresentaram hábitos nocivos.

Os hábitos de sucção não-nutritivos foram bastante comuns entre as crianças pesquisadas, estando, portanto, de acordo com o relatado na literatura ${ }^{8,24}$. No entanto, frequências menores foram encontradas por Warren et al. ${ }^{16}$ e Paunio et al. ${ }^{22}$, com $20,0 \%$ e $25,1 \%$, respectivamente.
Tabela 4. Avaliação dos hábitos de sucção não-nutritivos segundo o tempo de aleitamento natural exclusivo e aleitamento natural total no grupo de crianças com aleitamento natural exclusivo . João Pessoa (PB).

\begin{tabular}{|c|c|c|c|c|c|c|}
\hline \multirow[t]{3}{*}{ Tempo em meses } & \multicolumn{4}{|c|}{$\begin{array}{c}\text { Hábitos de sucção } \\
\text { não-nutritivos }\end{array}$} & & \\
\hline & \multicolumn{2}{|c|}{ Sim } & \multicolumn{2}{|c|}{ Não } & \multicolumn{2}{|c|}{ Total } \\
\hline & $\mathrm{n}$ & $\%$ & $n$ & $\%$ & $\mathrm{n}$ & $\%$ \\
\hline \multicolumn{7}{|l|}{$\begin{array}{l}\text { Aleitamento } \\
\text { natural exclusivo }\end{array}$} \\
\hline Até 6 & 3 & 16,7 & 15 & 83,3 & 18 & 100,0 \\
\hline M ais de 6 até 12 & - & - & & 100,0 & 5 & 100,0 \\
\hline M ais de 12 a 24 & 2 & 33,3 & 4 & 66,7 & 6 & 100,0 \\
\hline Total & 5 & 17,2 & 24 & 82,8 & 29 & 100,0 \\
\hline \multicolumn{7}{|l|}{$\begin{array}{l}\text { Aleitamento } \\
\text { natural total }\end{array}$} \\
\hline Até 6 & 1 & 25,0 & 3 & 75,0 & 4 & 100,0 \\
\hline M ais de 6 até 12 & 1 & 50,0 & 1 & 50,0 & 2 & 100,0 \\
\hline M ais de 12 a 24 & 2 & 14,3 & 12 & 85,7 & 14 & 100,0 \\
\hline M ais de 24 a 36 & 1 & 11,1 & 8 & 88,9 & 9 & 100,0 \\
\hline Total & 5 & 17,2 & 24 & 82,8 & 29 & 100,0 \\
\hline
\end{tabular}

Tabela 5. Avaliação dos hábitos de sucção não-nutritivos segundo o tempo de aleitamento natural exclusivo, aleitamento natural total e aleitamento artificial no grupo de crianças com aleitamento misto. João Pessoa (PB).

\begin{tabular}{|c|c|c|c|c|c|c|c|c|}
\hline \multirow[t]{3}{*}{ Tempo em meses } & \multicolumn{4}{|c|}{$\begin{array}{l}\text { Hábitos de sucção } \\
\text { não-nutritivos }\end{array}$} & & & \multirow[t]{3}{*}{ Valor de P } & \multirow[t]{3}{*}{ OR E IC com $95,0 \%$} \\
\hline & \multicolumn{2}{|c|}{ Sim } & \multicolumn{2}{|c|}{ Não } & \multicolumn{2}{|c|}{ Total } & & \\
\hline & $n$ & $\%$ & $n$ & $\%$ & $n$ & $\%$ & & \\
\hline \multicolumn{9}{|l|}{$\begin{array}{l}\text { Aleitamento } \\
\text { natural exclusivo }\end{array}$} \\
\hline Até 6 & 164 & 79,3 & 43 & 20,8 & 207 & 100,0 & & \\
\hline$M$ ais de 6 até 12 & 15 & 51,7 & 14 & 48,3 & 29 & 100,0 & $\mathrm{P}^{2}<0,001^{*}$ & $* *$ \\
\hline M ais de 12 a 24 & 2 & 33,3 & 4 & 66,7 & 6 & 100,0 & & \\
\hline Total ${ }^{1}$ & 181 & 74,8 & 61 & 25,2 & 242 & 100,0 & & \\
\hline \multicolumn{9}{|l|}{$\begin{array}{l}\text { Aleitamento } \\
\text { natural total }\end{array}$} \\
\hline Até 6 & 143 & 87,2 & 21 & 12,8 & 164 & 100,0 & & \\
\hline M ais de 6 até 12 & 24 & 68,6 & 11 & 31,4 & 35 & 100,0 & $\mathrm{P}^{3}<0,001^{*}$ & $* *$ \\
\hline M ais de 12 a 24 & 8 & 28,6 & 20 & 71,4 & 28 & 100,0 & & \\
\hline M ais de 24 a 36 & 6 & 40,0 & 9 & 60,0 & 15 & 100,0 & & \\
\hline Total 1 & 181 & 74,8 & 61 & 25,2 & 242 & 100,0 & & \\
\hline \multicolumn{9}{|l|}{$\begin{array}{l}\text { Aleitamento } \\
\text { artificial }\end{array}$} \\
\hline Até 6 & 2 & 28,6 & 5 & 71,4 & 7 & 100,0 & & $0,11(0,01$ a 0,58$)$ \\
\hline$M$ ais de 6 até 12 & 24 & 75,0 & 8 & 25,0 & 32 & 100,0 & $P^{3}=0,026^{*}$ & $0,79(0,32$ a 1,98$)$ \\
\hline M ais de 12 a 24 & 64 & 72,7 & 24 & 27,3 & 88 & 100,0 & & $0,70(0,37$ a 1,34$)$ \\
\hline M ais de 24 a 36 & 91 & 79,1 & 24 & 20,9 & 115 & 100,0 & & 1,00 \\
\hline Total ${ }^{1}$ & 181 & 74,8 & 61 & 25,2 & 242 & 100,0 & & \\
\hline
\end{tabular}

(*) Associação significante ao nível de 5,0\%; (**) N ão foi determinado devido à presença de frequência muita baixa. (1) Para sete pesquisados, não se dispõe das informações sobre amamentação; (2) Através do teste Exato deFisher; (3) Através do testequi-quadrado. 
Os hábitos de sucção não-nutritivos podem ter origem emocional, causado pela falta de afeto materno; podem ser imitativos, em que as crianças aprendem o hábito por imitação dos atos dos pais, amigos ou irmãos pequenos; e podem originar-se através das primeiras experiências de alimentação, nas quais os padrões iniciais pouco adequados predispõem a formação dos maus hábitos, como, por exemplo, alimentação muito rápida, quantidade insuficiente de comida em cada mamada e tensão da mãe no momento da amamentação ${ }^{11,16,24,25}$.

A maioria das crianças iniciou o hábito de suç̧ão não-nutritivo no período desde o nascimento até os três primeiros meses de vida, concordando com outros estudos similares ${ }^{4,19}$. A prevalência de sucção de chupeta verificada no presente trabalho $(61,6 \%)$ está em concordância com a literatura, cujos resultados situam-se entre $21,0 \%^{8}$ a $72,0 \%^{20}$. Crianças que possuem 0 hábito de sucção de chupeta mostram maior prevalência de alterações oclusais nas relações dos arcos dentais e das estruturas miofuncionais quando comparadas àquelas que não possuem este tipo de hábito ${ }^{10}$.

Com relação à sucção digital, os resultados

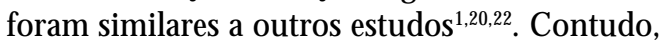
vários autores obtiveram resultados diferentes, sendo a prevalência da sucção digital superior à da sucção de chupeta, em valores que variam de $24,0 \%$ a 54,9\% 8,16,21,23,25,26. Essa discrepância entre os estudos pode, provavelmente, ser explicada pelas características individuais da população estudada, a faixa etária envolvida e o número de sujeitos pesquisados.

0 maior percentual de crianças permaneceu com os hábitos de sucção não-nutritivos (sucção digital e de chupeta) atéo período de 25 a 36 meses $(62,5 \%)$. Todavia, esse resultado está em discordância com os obtidos por Warren et al. ${ }^{16}$ e Solis et al. ${ }^{25}$, os quais verificaram que as crianças abandonaram o hábito até os doze meses de idade (31,9\% e $85,6 \%$, respectivamente).

A análise da frequência diurna e noturna do hábito de sucção de chupeta revelou que muitas das crianças utilizavam-na nos períodos diurno e noturno. Entretanto, com relação ao tipo de chupeta utilizado, a prevalência de crianças que usaram chupeta tradicional foi superior ao reportado na literatura ${ }^{1,4,21}$ e apenas $17,8 \%$ da amostra fizeram uso da chupeta ortodôntica. Pode-se inferir que talvez a falta de informação das mães, associada à ausência de correta orientação pelos profissionais de saúde que acompanham essas crianças - sejam eles pediatras, ci- rurgiões-dentistas e outros - possam ter contribuído para essa prática. Ademais, o custo das chupetas tradicionais ainda é menor, fazendo com que a escolha da chupeta seja mais uma questão de ordem financeira.

Considerando o número de crianças que tiveram aleitamento natural exclusivo, apesar dea frequênciater sido baixa (10,2\%), mostra-secompatível com outros estudos ${ }^{19,21,27}$. Por sua vez, 0 al eitamento artificial exclusivo foi encontrado em apenas $4,9 \%$ da amostra, sendo esse resultado inferior ao reportado por outros autores ${ }^{19,21,23,28}$, cuja frequência varia de 10,0\% a 59,7\%.

Encontrou-seuma elevada prevalência decrianças com aleitamento misto (84,9\%); porém, a literatura reporta frequências inferiores que variam de $17,07 \%$ a $37,2 \%{ }^{19,21,28}$.

Considerando a amostra total, verifica-seque $83,3 \%$ das crianças que realizaram o al eitamento natural exclusivo o fizeram até os seis primeiros meses de vida; $12,0 \%$ o realizaram até o período de seis meses a doze meses e $4,2 \%$, até o período de mais de doze a 24 meses. A duração média do aleitamento natural exclusivo foi de 4,6 meses. Com relação ao tempo de aleitamento natural total, observa-se que $63,9 \%$ o realizaram até os seis meses, diminuindo esta prevalência até o período de mais de 24 a 36 meses $(8,4 \%)$.

Segundo a Organização Mundial de Saúde², o leite materno deve ser oferecido de maneira exclusiva durante os seis meses de vida - podendo ser prolongado até os dois anos de idade desde que os processos de crescimento e desenvolvimento estejam evoluindo dentro dos parâmetros normais. Portanto, pode-se afirmar que as crianças apresentaram padrão de aleitamento compatível com o preconizado, visto que um número expressivo $(83,8 \%)$ realizou o al eitamento natural exclusivo durante os seis primeiros meses de vida.

Este estudo revelou que, dentre as crianças com aleitamento natural exclusivo, a maioria não tinha nenhum tipo de hábito de sucção não-nutritivo, corroborando o descrito por estudos pré vios $^{18,22}$, observando que quanto mais tempo foi realizado 0 aleitamento natural, menor a prevalência de hábitos de sucção.

O percentual da ocorrência dehábitos desucção não-nutritivos diminuiu à medida queo tempo de amamentação exclusiva aumentou. No entanto, Shoaf ${ }^{23}$ observou que as crianças al eitadas no seio apresentaram maior prevalência de hábitos de sucção do que as crianças que realizaram al eitamento artificial. Em contrapartida, as primeiras apresentavam menor duração do hábito. 
Em relação à amamentação artificial, o me nor percentual ocorreu na faixa até seis meses, quetinha setecrianças $(28,6 \%$ ), evariou de $72,7 \%$ a $79,1 \%$ nas outras três faixas, ou seja, quanto mais tempo a mamadeira foi usada como forma de aleitamento, maior a prevalência de hábitos. A associação entre as faixas de tempo de aleitamento e a ocorrência de hábitos de sucção nãonutritivos é significante, demonstrando que as crianças cujo aleitamento natural foi predominante provavelmente tiveram a sua necessidade de sucção sati sfeita de forma mais completa, necessitando menos de outros artifícios, como o dedo ou a chupeta, para a complementação desta necessidade.

As crianças aleitadas com mamadeira por mais de um ano apresentam quase dez vezes mais risco de apresentarem hábitos bucais viciosos do que aquelas que nunca utilizaram essa forma de al eitamento ${ }^{12}$. Ademais, o uso da mamadeira podeinterferir negativamenteno desenvolvimento orofacial ${ }^{29}$.

A relação entre a duração dos hábitos de sucção não-nutritivos e os tempos de aleitamento foi confirmada neste estudo. Diante dos resultados, pode-se perceber a necessidade de implantação de programas de promoção e o incentivo ao aleitamento natural como forma de alimentação de bebês, o qual se mostrou bastante importante na prevenção da instalação de hábitos de sucção não-nutritivos que possam comprometer o desenvolvimento normal do sistema estomatognático.

\section{Colaboradores}

SSL Albuquerque M oura realizou a coleta de dados, análise, interpretação e discussão dos mesmos e redação do artigo científico. RC Duarte, na qualidade de orientador, participou do delineamento metodológico, da discussão dos dados e da redação do artigo. AL Cavalcanti contribuiu na análise, discussão e interpretação dos resultados, redação e revisão final do artigo. EM Beltrão colaborou na coleta de dados e redação do artigo. 
1. Legovic $M$, Ostric $L$. The effects of feeding methods on the growth of the jaws in infants. ASDC J Dent Child 1991; 58(3):253-255.

2. Organização Mundial de Saúde. Alimentação infantil: bases fisiológicas. Genebra: OM S; 1989.

3. Turgeon-O'Brien H, Lachapelle D, Gagnon PF, Larocque I, Maheu-Robert LF. Nutritive and nonnutritive sucking habits: A review. ASDC J Dent Child 1996; 63(5):321-327.

4. Adair SM, Milano M, Dushku JC. Evaluation of the effects of orthodontic pacifiers on the primary dentitions of 24- to 59-month-old children: preliminary study. Pediatr Dent 1992; 14(1):13-18.

5. Aznar T, Galán AF, Marín I, Domínguez A. Dental arch diameters and relationships to oral habits. Angle Orthod. 2006; 76(3):441-445.

6. Poyac J. Effects of pacifiers on early oral development. Int J Orthod M ilwaukee 2006; 17(4):13-16.

7. Ganesh M, Tandon S, Sajida B. Prolonged feeding practice and its effects on developing dentition. J Indian Soc Pedod Prev Dent 2005; 23(3):141-145.

8. López Del Valle LM, Singh GD, Feliciano N, Machuca $M$ del $C$. Associations between a history of breast feeding, malocclusion and parafunctional habits in Puerto Rican children. P R Health Sci J 2006; 25(1):31-34.

9. Viggiano D, Fasano D, Monaco G, Strohmenger $L$. Breast feeding, bottle feeding, and non-nutritive sucking; effects on occlusion in deciduous dentition. Arch Dis Child. 2004; 89(12):1121-1123.

10. Zardetto CG, Rodrigues CR, Stefani FM. Effects of different pacifiers on the primary dentition and oral myofunctional structures of preschool children. Pediatr Dent 2002; 24(6):552-560.

11. Aravena P, Alvarez F, Roizen S. Efecto del mal hábito de succión en la dentición en niños. Pediatr día 1998; 14(5):269-272.

12. Serra-Negra JM C, Pordeus IA, Rocha Jr JF. Estudo da associação entre aleitamento, hábitos bucais e maloclusões. Rev Odontol U niv São Paulo 1997; $11(2): 79-86$.

13. Caglar E, Larsson E, Andersson EM, Hauge MS, Ogaard B, Bishara S, Warren J, Noda T, Dolci GS. Feeding, artificial sucking habits, and malocclusions in 3-year-old girls in different regions of the world. J Dent Child 2005; 72(1):25-30.

14. Monguilhott LMJ, Frazzon JS, Cherem VB. Hábitos de Sucção: como e quando tratar na ótica Ortodontia x Fonoaudiologia. Rev Dent Press Ortod Ortoped Facial 2003; 8(1):95-104.

15. Johnson ED, Larson BE. Thumb-sucking: Classification and treatment. ASDC J Dent Child 1993; 60(4):392-398.

16. Warren JJ, Bishara SE, Steinbock KL, Yonezu T, N owak AJ. Effects of oral habits' duration on dental characteristics in the primary dentition. J Am Dent Assoc 2001; 132(12):1685-1693.
17. Silva Filho OG, Gonçalves RM G, M aia FA. Sucking habits: clinical management in dentistry. J Clin Pe diatr Dent 1991; 15(3):137-156.

18. Aarts C, Hornell A, Kylberg E, H ofvander Y, Gebre$M$ edhin $M$. Breastfeeding patterns in relation to thumb sucking and pacifier use. Pediatrics 1999; 104(4):e50.

19. Hanna JC. Breast feeding versus bottle feeding in relation to oral habits. ASDC J Dent Child 1967; 34(4):243-249.

20. Larson E. Sucking, chewing and feeding habits and the development of crossbite: A longitudinal study of girls from birth to 3 years of age. Angle Orthod 2001; 71(2):116-119.

21. M eyers A, Hertzberg J. Bottle-feeding and malocclusion: Is there an association? Am J Orthod Dentofacial Orthop 1988; 93(2):149-152.

22. Paunio P, Rautava P, Sillanpaa M. The Finnish Family Competence Study: The effects of living conditions on sucking habits in 3-year-old Finnish children and the association between these habits and dental occlusion. Acta Odontol Scand 1993; 51:23-29.

23. Shoaf HK. Prevalence and duration of thumbsucking in breast-fed and bottle-fed children. ASDC J Dent Child 1979; 46(2):126-129.

24. Bayardo RE, M ejia JJ, Orozco S, M ontoya K. Etiology of oral habits. ASDC J Dent Child 1996; 63(5):350-353.

25. Solis CEM, Rosado JFC, Rosado AJC. M alos habitos orales en infantes de guarderías del IMSS. Rev M ed IM SS 2001; 39(5):435-440.

26. Infante PF. An epidemiologic study of finger habits in preschool children, as related to maloclusion, socioeconomic status, race, sex, and size of community. ASDC J Dent Child 1976; 43(1):33-38.

27. Lande B, Andersen LF, Baerug A, Trygg KU, LundLarsen K, Veierød M B, Bjørneboe GE. Infant feeding practices and associated factors in the first six months of life: the Norwegian infant nutrition survey. Acta Paediatr 2003; 92(2):152-161.

28. Senarath U, Dibley MJ, Agho KE. Breastfeeding practices and associated factors among children under 24 months of age in Timor-Leste. Eur J Clin Nutr. 2007; 61(3):387-397.

29. Carrascoza KC, Possobon Rde F, Tomita LM, Moraes $A B$. Consequences of bottle-feeding to the oral facial development of initially breastfed children. J. Pediatr. 2006; 82(5):395-397.

Artigo apresentado em 11/07/2007

Aprovado em 14/12/2007

Versão final apresentada em 16/01/2008 